\title{
Los blogs como entornos virtuales de enseñanza y aprendizaje en Educación Superior ${ }^{1}$
}

\section{Blogs as virtual environments for teaching and learning in Higher Education}

\author{
Pere MOLINA ALVENTOSA ${ }^{1}$, Javier VALENCIANO VALCÁRCEL ${ }^{2}$ y \\ Alexandra VALENCIA-PERIS ${ }^{1}$ \\ ${ }^{1}$ Universitat de València y ${ }^{2}$ Universidad de Castilla La Mancha
}

Recibido: Diciembre 2013

Aceptado: Abril 2014

\begin{abstract}
Resumen
En la actualidad, existe un interés pedagógico por transformar las tecnologías de la información y comunicación en tecnologías para el aprendizaje y el conocimiento. En este trabajo se presentan una serie de contribuciones didácticas asociadas al uso de blogs en la Educación Superior a partir de una experiencia de innovación educativa por proyectos llevada a cabo por un grupo de profesores universitarios entre los años 2009 y 2013. Tanto el profesorado como el alumnado participante en las asignaturas donde se ha puesto en práctica el edublog, lo verifican como un recurso útil para conformar entornos virtuales de enseñanza y aprendizaje gracias a las múltiples potencialidades que ofrece. Entre ellas se destacan: facilitar el acceso al conocimiento, promover una participación más activa y reflexiva en la construcción de los aprendizajes, amplificar la experiencia social del aprendizaje, proporcionar evidencias sobre el progreso de los estudiantes que ayuden a reorientar el proceso de enseñanza-aprendizaje y estimular el juicio crítico. No obstante, también se identifican problemas relacionados con la participación de los estudiantes y con la gestión de los edublogs por parte del profesorado.
\end{abstract}

Palabras clave: innovación pedagógica, tecnologías de la información y de la comunicación, uso didáctico del ordenador, enseñanza superior.

\begin{abstract}
The transformation of the Information and Communication Technologies (ICT) into knowledge and learning technologies is increasingly becoming a matter of concern. Teaching settings associated with the use of blogs in Higher Education are presented in this paper, proceeding from an innovative learning experience of projects carried out by a group of professors between 2009 and 2013. Both, teachers and students who took part in the subjects that implemented the blog, considered it as helpful resource to create a virtual and learning-teaching environment due to the multiple potentialities it offers. Among some of these potentialities, some stand out: it
\end{abstract}

\footnotetext{
${ }^{1}$ Este trabajo ha sido parcialmente financiado por la Universitat de València a través de sus convocatorias a proyectos de innovación educativa para los cursos 2009-10, 2010-11, 2011-12 y 2012-13.
} 
makes easier the access to knowledge, promotes a more active and reflective learning, expands the social experience of learning, provides evidence about the students' progress which helps to reorient the teaching-learning process, and encourages the critical judgment. Nevertheless, several problems related with the students' participation and the teacher's blog management have been identified.

Keywords: educational innovation, information and communication technologies, didactic use of computer, group learning, higher education.

Las tecnologías de la información y comunicación (TIC) se han introducido en las últimas décadas en todos los ámbitos del mundo desarrollado donde se llevan a cabo procesos de enseñanza-aprendizaje. En general, este tipo de tecnología educativa ha facilitado estos procesos tanto desde la perspectiva del que se propone enseñar como del que pretende aprender. Nadie podría negar que enseñar y aprender es hoy una tarea mucho más accesible gracias a las TIC, las cuales han evolucionado a un ritmo vertiginoso, en especial, desde la aparición de Internet y, más concretamente, la World Wide Web (www). Desde los sistemas de composición y proyección audiovisual, que amplificaron las posibilidades de la pizarra, hasta las aplicaciones sociales de la Web 2.0, los recursos que las TIC han puesto al servicio de la educación han experimentado toda una revolución. Hasta el punto de que, en muchas ocasiones, sus nuevos dispositivos tecnológicos, aplicaciones o utilidades se han introducido en la praxis educativa antes incluso de haber sido abordados por la teoría pedagógica (García y Suárez, 2011; O’Donnell, 2006) y debemos reconocer que, a pesar de la gran capacidad que tienen las TIC en el tratamiento de la información, su mera incorporación no mejora necesaria y automáticamente los procesos de enseñanza-aprendizaje (Coll y Monereo, 2008).

En este sentido, existe una gran preocupación pedagógica por orientar las TIC hacia usos más formativos, al servicio del aprendizaje y la adquisición de conocimiento, incidiendo especialmente en la metodología de enseñanza, más que en el dominio del funcionamiento de los dispositivos tecnológicos y sus utilidades. La propuesta consiste en transformar las TIC en tecnologías para el aprendizaje y el conocimiento (TAC). A este respecto, Coll (2004) recuerda que el acceso a la información da lugar al aprendizaje y la información se convierte en conocimiento cuando somos capaces de darle significado y sentido. Sancho (2008), por su parte, advierte que esta transformación debe ser enmarcada dentro de un contexto educativo, con unas finalidades y un sistema de seguimiento que nos permita valorar educativamente la integración de las TIC como experiencia de aprendizaje.

Asimismo, Bustos y Coll (2010) señalan que la integración de las TIC en el ámbito educativo debe significar también la transformación de los entornos tradicionales y la creación de nuevos entornos de enseñanza y aprendizaje. Estos autores destacan especialmente los que se basan en configuraciones construidas sobre las posibilidades de interconexión e intercomunicación que ofrecen las TIC, es decir, los entornos virtuales o en línea. Uno de los entornos virtuales como espacio de enseñanza y aprendizaje es el que se genera a través del uso del blog, que en el ámbito de la educación suelen denominarse edublogs. 
El blog es un recurso ampliamente utilizado en el ámbito de la Educación Superior, en sus diferentes ramas de conocimiento y en el contexto nacional e internacional (Cabero, López y Ballesteros, 2009; Gallego, Martín-Cuadrado y Cacheiro, 2010; Sim y Hew, 2010; Williams y Jacobs, 2004). La popularización de las aplicaciones didácticas de los blogs se debe probablemente a su sencillez frente a la edición de páginas web, su interactividad y la posibilidad de añadir contenidos multimedia o enlazar otras fuentes de información. Además de estas prestaciones técnicas, y a diferencia de otras herramientas de la web social esencialmente monológicas, Fernández (2010) destaca el carácter dialógico de los blogs. Aunque en las redes sociales como Facebook o Twitter pueda producirse el diálogo, éste no resulta tan esencial como pueda serlo en un blog. El blog persigue deliberadamente generar debate $\mathrm{y}$, para ello, tiene herramientas que permiten estructurar y enlazar la información para darle cierto orden y que los temas puedan ser abordados en profundidad y desde diferentes perspectivas.

Pero en última instancia, la introducción de este recurso en la Educación Superior debería basarse fundamentalmente en su repercusión sobre los aprendizajes. Además de que los blogs ayuden a la comprensión de los temas en cuestión y a consolidar los conocimientos (Coutinho, 2007; Ellison y Wu, 2008), la investigación les atribuye experiencias formativas que facilitan el desarrollo de diversas capacidades de interés en la enseñanza universitaria. En estos espacios virtuales se proporcionan diferentes puntos de vista y se fomenta el intercambio y el debate intelectual (William y Jacobs, 2004; Xie y Sharma, 2005; Zeng y Harris, 2005). Permiten un pensamiento y una escritura más reflexivos, dando lugar a discursos más estructurados (Lara, 2005; Xie, Ke y Sharma, 2008) y, por otra parte, desarrollan habilidades para el trabajo colaborativo (García y Suárez, 2011; Lara, 2005).

Aun así, debido a las limitaciones metodológicas de los estudios, algunos autores consideran que la utilidad de los blogs para la mejora de los aprendizajes y de las capacidades intelectuales es todavía una cuestión por resolver (Sim y Hew, 2010). Entre otras cosas, consideran insuficiente el marco temporal de los mismos, reducidos en muchos casos a un semestre del curso, y demandan estudios a más largo plazo para evaluar los efectos del uso continuado de los blogs.

Con el fin de contribuir a esclarecer esta cuestión, en este trabajo se presentan las principales contribuciones didácticas que los blogs pueden aportar a la creación de entornos virtuales para la enseñanza y aprendizaje en la Educación Superior, a partir de la experiencia acumulada en el uso de esta TIC dentro la práctica docente de un grupo de innovación educativa durante cuatro cursos académicos.

\section{El uso de los edublogs en Educación Superior a través del desarrollo de proyectos de innovación educativa}

Las aportaciones que se presentan en este trabajo provienen de diversos proyectos de innovación que se gestaron entre varios profesores de la Universitat de València (UV). Tomando como antecedente la experiencia previa de alguno de ellos, a partir del curso 2009-10 y ante el inicio de la implantación de los estudios de Grado, estos 
profesores decidieron constituirse como Grupo de Innovación Educativa (GID) y concurrir a las convocatorias del Servicio de Formación Permanente e Innovación Educativa de la UV.

El primero de los proyectos (2009-10) tuvo por objeto el diseño e implementación de edublogs en varias asignaturas de la todavía licenciatura en Ciencias de la Actividad Física y el Deporte. Se experimentaron las posibilidades de este recurso caracterizando diversos tipos, metodologías y usos didácticos de los blogs en la docencia universitaria en función de su contribución al desarrollo de competencias relacionadas con el análisis y el tratamiento de la información y con la utilización de las TIC (Antolín, Molina, Villamón, Devís y Pérez-Samaniego, 2011).

El segundo proyecto (2010-11) se dedicó a estudiar la utilidad de los blogs para la evaluación continua de los aprendizajes. En esta ocasión, el grupo de innovación se vio ampliado al contar con profesorado de la Universidad de Castilla La Mancha (UCLM). Esta participación supuso extender el proyecto a otras enseñanzas, en concreto, al Grado en Maestro en Educación Primaria de la UCLM. Este proyecto sirvió para definir formas de evaluación continuada del aprendizaje según el tipo y características del blog utilizado en la asignatura y para relacionar las formas de evaluación del aprendizaje del alumnado definidas con el desarrollo de competencias vinculadas con el análisis y tratamiento de la información, y con la utilización de las TIC por parte del alumnado. Asimismo, en esta ocasión se incorporaron procesos de evaluación de la experiencia, a partir de las opiniones de los docentes y del alumnado (Molina, Antolín, Pérez-Samaniego et al., 2013).

El tercer proyecto (2011-12) se centró en el uso de los blogs como recurso para la participación y seguimiento de actividades académicas complementarias y transversales y el desarrollo de competencias instrumentales, en las mismas asignaturas y enseñanzas que en el proyecto anterior. Dos de los blogs más representativos de este proyecto fueron una revista-blog, administrada conjuntamente por el profesorado y los estudiantes, con proceso de revisión editorial incluido ${ }^{2}$ y un blog para el seguimiento de un seminario de formación permanente conformado por una serie de actividades complementarias dirigidas a la participación de los estudiantes. Estas actividades tenían un carácter presencial, pero eran filmadas por diferentes grupos de estudiantes que elaboraban vídeos-resúmenes para, posteriormente, colgarlos en Youtube e insertarlos como posts en el blog. Así estos posts eran una prolongación no presencial de participación virtual e interactiva entre los estudiantes en las actividades llevadas a cabo (Molina, Antolín, Devís et al., 2013).

En el cuarto proyecto (2012-13) el GID ya consolidado se constituyó como una red de innovación integrando a profesorado de más universidades y titulaciones, configurándose un grupo interdisciplinar de 28 profesores y profesoras de 5 universidades españolas. Además de la UV y la UCLM, participaron la Universidad

\footnotetext{
${ }^{2}$ La revista-blog obtuvo el reconocimiento de la UV, a través de su programa ESTIC, dirigido a instaurar modelos de aprendizaje y estudio colaborativo entre el alumnado, así como el uso y aplicación de TIC en el ámbito universitario, y el $3^{\text {er }}$ premio en la categoría de 'Blogs de Formación de Personas Adultas, Escuelas Oficiales de Idiomas y Universidad’, en la VI edición de los premios Espiral de edublogs.
} 
Cardenal Herrera-CEU de Valencia, la Universidad Miguel Hernández y la Universidad del País Vasco y, del mismo modo, se incorporaron asignaturas de los Grados en Sociología, en Periodismo, en Comunicación Audiovisual y en Maestro en Educación Infantil. En este proyecto los blogs fueron utilizados para incidir en el aprendizaje colaborativo (Molina, Valenciano et al., 2013).

\section{Metodología}

Para el seguimiento de los proyectos de innovación llevados a cabo y de sus resultados se recurrió, como metodología general de trabajo, al seminario colaborativo y a la administración de encuestas a profesores y estudiantes. El seminario colaborativo se entiende como una técnica activa de enseñanza e investigación basada en el trabajo en grupo e intercambio de información, utilizada para profundizar desde el debate y el análisis colectivo en un tema determinado (Fraile, 2002). A lo largo de todos estos proyectos se celebraron sesiones periódicas para poner en común las experiencias que estaban desarrollando los profesores participantes. En el caso de las encuestas, las dirigidas al profesorado, combinaban cuestiones con respuestas estructuradas mediante diferentes opciones y otras de respuesta abierta, donde se pide al encuestado que sea él mismo quien aporte el contenido de su contribución. Entre otros aspectos, se les preguntaba sobre los motivos que les habían impulsado a poner en marcha un blog, cuál era el principal uso que hacían del mismo, qué tipo de actividades desarrollaban, qué ventajas y problemas identificaban y su grado de satisfacción en relación con esta herramienta. Las encuestas dirigidas al alumnado fueron de respuesta abierta y se administraron al final de algunas experiencias. En unas de ellas, se solicitaba, desde un post del edublog de la asignatura, que hiciesen una valoración de la experiencia desde su punto de vista, señalando aspectos positivos y negativos y aportando alguna propuesta de mejora; en otras experiencias, también desde un post del blog docente, se invitaba al alumnado a elaborar una autoevaluación de su aprendizaje en la asignatura mediante sus comentarios al post del profesor o elaborando un post específico en los respectivos blogs de los alumnos.

Las deliberaciones y conclusiones del seminario colaborativo durante los cuatro cursos y los datos procedentes de las diversas encuestas han constituido la base a partir de la cual responder al propósito de este artículo. En cuanto a las encuestas al alumnado, concretamente, se contemplaron opiniones realizadas por escrito de 91 estudiantes (que participaron en alguna de las experiencias durante los cuatro cursos académicos) y las contestaciones a la encuesta de 16 profesores que participaron en el cuarto proyecto. El análisis consistió en identificar unidades temáticas a partir de las opiniones manifestadas en los blogs por parte del estudiantado, para indagar de qué manera se correspondían con las percepciones señaladas por el profesorado a través de las encuestas y los seminarios llevados a cabo. 


\section{La conformación de entornos virtuales de enseñanza y aprendizaje a través de los edublogs}

En este apartado se exponen las bondades atribuidas al uso de los edublogs para favorecer la conformación de entornos virtuales de enseñanza y aprendizaje en Educación Superior, a partir de la experiencia acumulada por el grupo de innovación en los proyectos reseñados con anterioridad. En primer lugar, como los blogs son variopintos y sus características pueden ayudar a comprender mejor su uso y utilidad, se presentan las posibilidades de uso experimentadas por el grupo de innovación.

\section{Posibilidades de participación e interactividad de los edublogs}

Los blogs utilizados a lo largo de estos años varían entre sí debido a razones diversas como la naturaleza teórica o práctica de las asignaturas, el tipo de actividades formativas relacionadas con el blog, los fines con que era utilizado o, en general, el papel principal o secundario que este recurso representaba entre las diferentes estrategidas didácticas de cada asignatura. Mejor que la descripción de cada uno de ellos, que resultaría poco operativa, se muestra una clasificación de los mismos, basada en otros trabajos (Antolín et al., 2011; Valencia-Peris y Molina, 2012), de acuerdo al grado de participación e interactividad que posibilitan:

a) El blog docente como transmisor de la información de la asignatura

En este caso el edublog se utiliza como un espacio virtual donde el profesorado aporta a los estudiantes información relacionada con los contenidos o el desarrollo de la asignatura. El grado de comunicación y de intercambio entre los propios estudiantes y el profesorado que se recoge dentro del blog es mínimo.

\section{b) El blog abierto a la participación del alumnado a través de sus comentarios}

Este tipo de uso de edublogs se caracteriza por tener al profesorado como autor y administrador del blog y ser quien elabora de forma frecuente los posts que posteriormente comentará el alumnado. Esta metodología ofrece muchas posibilidades aumentando notablemente la participación del alumnado, promoviendo el intercambio de información y el aprendizaje colaborativo.

c) El blog abierto a la participación del alumnado a través de sus posts

En esta tipología de uso de blogs destaca el hecho de que, aparte de ser el profesorado el encargado de realizar los posts, los estudiantes también pueden elaborar y publicar entradas en las mismas condiciones que sus profesores, es decir, el alumnado es co-autor del blog.

\section{d) Los alumnos como administradores de sus blogs}

En este tipo de uso son los propios estudiantes los que se convierten en administradores de sus edublogs, tomando decisiones sobre el contenido y formato de los posts, del diseño y de la configuración de los mismos, etc. Cabe diferenciar aquí dos formas de uso del alumnado como administrador de sus blogs: a) el blog individual del alumno, que sirve para recoger el trabajo del alumno en la asignatura 
o como diario o cuaderno personal donde refleja reflexiones y comentarios propios del proceso de aprendizaje; y b) el blog grupal de los alumnos, que en este caso refleja el trabajo realizado en equipo. En esta última opción se almacena y muestra el trabajo hecho por el grupo pero, en este caso, no hay conexión ni red educativa virtual que muestre el resto de blogs elaborados por otros grupos de trabajo, por lo que no hay comunicación inter-grupal, como ocurre en las experiencias que se presentaran en el siguiente epígrafe.

\section{e) Los blogs como redes de aprendizaje o blogosferas educativas}

Con frecuencia, en aquellas asignaturas donde se hace un planteamiento metodológico para que cada alumno o grupo de alumnos creen un edublog, el profesorado establece una comunidad virtual o red de aprendizaje en formato de blogosfera educativa donde se vinculan los blogs de la asignatura. En ocasiones, para facilitar el seguimiento de los posts que aparecen en esta particular blogosfera educativa se utiliza un planeta de blogs o blogdeblogs donde aparecen vinculados el resto de blogs creados por los alumnos a través de hipervínculos o utilidades como los blogrolls que permiten ver en un mismo blog (administrado por el profesor, conjuntamente entre éste y el alumnado o por el propio alumnado) los últimos posts publicados de los blogs administrados por el alumnado de la asignatura. La diferencia entre este tipo de uso y los descritos en los apartados B, C y D es que mientras que en éstos existe una interacción intra-blog, que tiene lugar dentro de un solo blog, cuando se utiliza el blog como red de aprendizaje o blogosfera educativa, la interacción es inter-blogs, es decir, dentro de un conjunto de blogs.

En nuestros proyectos, cada tipo de uso integra el que le antecede (ver figura 1). Son cinco tipos de uso que van desde un uso no interactivo del blog hasta la mayor posibilidad de interacción que se consigue con la blogosfera educativa. En los tipos $b, c$ y $d$ la interacción se da intra-blog, es decir dentro de un solo blog, pero cuando se utiliza el blog como red de aprendizaje o blogosfera educativa, la interacción es además inter-blogs, es decir, dentro de un conjunto de blogs.

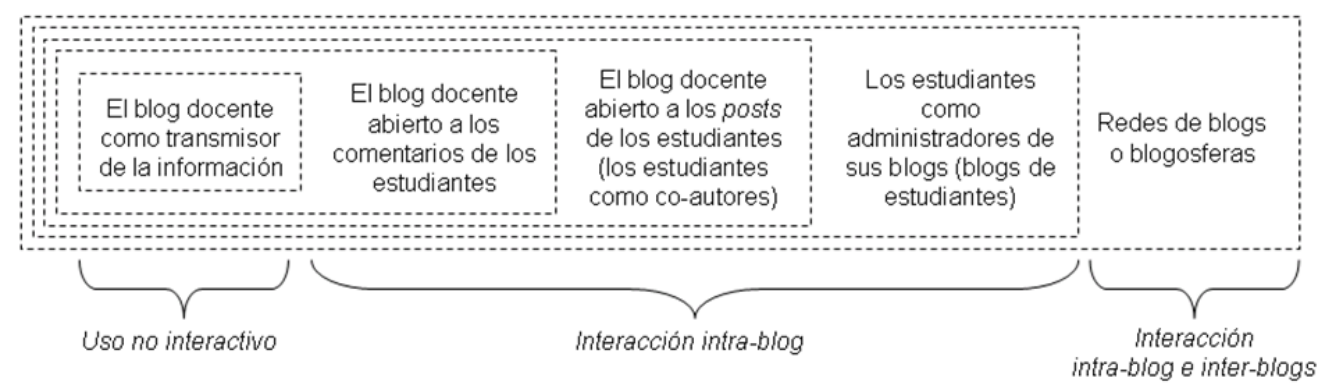

Figura 1. Tipos de uso de blogs y sus posibilidades de interacción.

A lo largo de estos años y entre todos los miembros del grupo de innovación, han sido utilizadas todas estas tipologías de uso de edublogs. No obstante, hay que señalar que el uso del blog tipo a ha sido minoritario, sólo se utilizó en los blogs de dos 
asignaturas del primer proyecto El resto de usos $(b, c, d$ y $e$ ), los cuales permiten la interacción de los estudiantes, son los que han predominado en nuestras experiencias. En el último proyecto, curso 2012-13, la mayor parte de las experiencias desarrolladas se encuadraban en los usos $c$ y $e$ (ver tabla 1$)$.

\begin{tabular}{|c|c|c|c|c|c|}
\hline & $\begin{array}{c}2009- \\
10\end{array}$ & $\begin{array}{c}2010- \\
11\end{array}$ & $\begin{array}{c}2011- \\
12\end{array}$ & $\begin{array}{c}2012- \\
13\end{array}$ & Totales \\
\hline $\begin{array}{l}\text { a) El blog docente como transmisor de la } \\
\text { información de la asignatura }\end{array}$ & 2 & - & - & - & 2 \\
\hline $\begin{array}{l}\text { b) El blog docente abierto a la participación del } \\
\text { alumnado a través de sus comentarios }\end{array}$ & 2 & 4 & 3 & 2 & 11 \\
\hline $\begin{array}{l}\text { c) El blog docente abierto a la participación del } \\
\text { alumnado a través de sus posts }\end{array}$ & 2 & 2 & 3 & 10 & 16 \\
\hline $\begin{array}{l}\text { d) Los alumnos como administradores de sus } \\
\text { blogs (el blog del alumno) }\end{array}$ & 2 & 2 & 3 & 1 & 8 \\
\hline $\begin{array}{l}\text { e) El blog como red de aprendizaje o blogosfera } \\
\text { educativa }\end{array}$ & - & - & 1 & 6 & 7 \\
\hline Totales: & 8 & 8 & 10 & 17 & 53 \\
\hline
\end{tabular}

Tabla 1. Número de experiencias desarrolladas en cada curso según el tipo de uso realizado del edublog.

\section{Potencialidades del edublog como entorno virtual de enseñanza y aprendizaje}

La experiencia acumulada por el grupo de innovación en el uso de edublogs y las evidencias obtenidas a lo largo de los diferentes proyectos permiten afirmar que este recurso ha favorecido el aprendizaje de los estudiantes universitarios que resultaron implicados. Tal es así que, en el último de los proyectos, el $75 \%$ del profesorado participante en la red de innovación utilizó el blog para actividades formativas que consideraban "fundamentales" para sus asignaturas y el 81\% acabó "satisfecho" o "muy satisfecho" con el resultado. En concreto, los aspectos para los cuales el blog ha supuesto una ayuda de interés para el aprendizaje son los que se comentan seguidamente.

\section{a) El blog facilita el acceso al conocimiento y un aprendizaje continuo}

El blog prolonga las oportunidades de aprender más allá del aula. Profesores y estudiantes, en función del tipo de blog, tienen opciones para retomar las cuestiones abiertas en el aula en ese espacio virtual para ampliarlas o profundizar en ellas. Esto permite reforzar los aprendizajes o sentar bases y realizar avances para construir los siguientes. Además, el blog permite desarrollar ideas paralelas o complementarias sobre los contenidos centrales de las asignaturas para los cuales, aun siendo de interés, no hay tiempo suficiente en las jornadas lectivas. Es una herramienta útil para incorporar o relacionar con el temario cuestiones de actualidad o novedades científicas no previstas de antemano en nuestras programaciones. Esta es una utilidad del blog que ha sido muy valorada por los estudiantes en otras ocasiones (Williams y Jacobs, 2004). 
Además de los comentarios que puedan efectuarse, es posible subir o enlazar materiales curriculares multimedia o de texto que enriquecen los apuntes personales de los estudiantes o la documentación básica de la asignatura. Esto puede hacerlo el profesor pero también los estudiantes y, en este caso último, las aportaciones y los materiales localizados están accesibles para todo el colectivo de clase, no solamente para sus redes personales. Las fuentes de información se diversifican así y dan más oportunidades para completar los aprendizajes iniciados en el aula de acuerdo a las necesidades y estilos de aprendizaje de cada cual:

Me decidí, para no ser egoísta con mis compañeros, a mostrar a través del blog todo aquello que me resultara interesante, dejar a un lado los miedos, (ies que todo lo que escriba lo van a leer!). El blog me parece la manera más eficaz en todos los aspectos, puesto que todos los contenidos que a través de él tenemos acceso sería casi imposible tenerlos (RSC $\left.{ }^{3}, 2012-13\right)$.

Por otra parte, si así desea enfocarlo el profesorado, el blog estimula una atención continua al desarrollo de la asignatura. Si las lecciones presenciales se conectan con las actividades del blog, éste se convierte también en un testigo de los progresos en la materia al que acudir para mantenerse al tanto de los avances. Éste es uno de los aspectos más valorados por los estudiantes y así lo han manifestado en diferentes ocasiones. Sirva como ejemplo este comentario de un alumno:

Por una parte, resulta un buen lugar de encuentro para los alumnos y el profesor alrededor de la asignatura y los temas que desarrolla. Esto resulta muy interesante porque este espacio es un lugar de referencia de la asignatura que complementa las clases teóricas y las prácticas que se dan en un espacio determinado y sólo durante un tiempo determinado (JE, 2009-10)

Si esto resulta de utilidad para cualquier estudiante, aquellos que, por diversas circunstancias, no pueden acudir a clase con regularidad todavía lo aprecian más. De acuerdo con Oravec (2003), los blogs pueden ocupar un espacio intermedio entre la enseñanza presencial y los sistemas de e-learning. Aunque el blog no esté pensado específicamente como un recurso para el seguimiento a distancia de las asignaturas, constituye un referente de suma utilidad para acceder al interior de la materia y orientar el aprendizaje autónomo de los estudiantes que han de optar por sistemas de evaluación alternativos al presencial.

b) El blog promueve una participación más activa y reflexiva en la construcción de los aprendizajes

\footnotetext{
${ }^{3}$ Con el objetivo de preservar el anonimato de las personas que han participado en la experiencia, los comentarios realizados por el alumnado se identifican mediante las iniciales de los mismos, mientras que los comentarios realizados por el profesorado se identifican con la letra P seguida de un número.
} 
En el marco de las metodologías activas, el profesorado ha de promover continuamente la implicación de los estudiantes en la construcción de sus propios aprendizajes. Entre otras cosas, se requiere que los estudiantes operen cognitivamente sobre los contenidos que han de aprehenderse. Es lo que los profesores intentan provocar a través de las preguntas y tareas que se suscitan en clase o se proponen para el tiempo de estudio o el trabajo autónomo. A lo largo de estos años, el blog se ha mostrado muy efectivo para este cometido, al igual que lo han percibido los estudiantes participantes en otras experiencias similares (Cabero et al., 2009; Williams y Jacobs, 2004).

Los estudiantes encuentran en el blog un espacio más confortable para lanzar sus intervenciones que en el contexto formal del aula, donde, en ocasiones y aun sin pretenderlo, la autoridad académica del profesor, el discurso dominante o el ritmo de la lección representan cortapisas implícitas para la participación de los estudiantes. La experiencia de los profesores ha corroborado que el blog resulta especialmente interesante para estimular la intervención de aquellos alumnos que, por sus características personales, permanecen invisibles durante las clases presenciales aun manteniendo una dedicación intensa a las asignaturas. Los profesores observaron que "el blog es un espacio de mayor libertad y permite al profesor conocer de modo distinto al alumno" (P10, 2012-13) y que "hay alumnado que en el aula no es elocuente (por timidez o por otras razones) y que encuentran aquí un lugar en el que realizar sus contribuciones de forma más cómoda" (P14, 2012-13). Aunque el espacio virtual no debiera sustituir el espacio físico del aula (García y Suárez, 2011), participar a través del blog puede constituir un primer paso para tomar la iniciativa y generar autoconfianza.

Al igual que han constatado otros estudios (Coutinho, 2007; Xie y Sharma, 2005), el blog da lugar a contribuciones de los estudiantes, en general, más elaboradas que las aportaciones que se producen en el ambiente natural de la clase. Los estudiantes tienen oportunidad de profundizar en los contenidos impartidos en las lecciones, de documentarse sobre ellos y de madurar y ordenar sus ideas, de modo que las aportaciones que introducen en el blog suelen tener un valor añadido con respecto a sus intervenciones en el aula. Los estudiantes lo han manifestado con este tipo de comentarios:

Y lo más importante, ha hecho que surtiera en mí el interés por aprender (por informarme para posteriormente poder hacer comentarios coherentes y documentados) hecho que sucede con poquísimas asignaturas a estas alturas de la carrera. (MM, 2010-11)

Además, el carácter abierto y estable del blog, al menos durante cada curso en vigor (así han sido los utilizados en estos proyectos), incentiva mayor esmero en los estudiantes al ser conscientes de la publicidad y permanencia de sus aportaciones. De esta manera, se promueve la responsabilidad pública de los estudiantes y su compromiso con la audiencia (Lara, 2005). 
En estos años se ha observado que el grado de elaboración de las aportaciones en los blogs alcanza también la expresión escrita de los estudiantes, tal y como han indicado otros autores (Lara, 2005; Neira, Villalustre y Moral, 2011; Zeng y Harris, 2005). El uso de blogs está relacionado con la promoción de la lectura y escritura, así como a la adquisición de habilidades de comunicación. Del mismo modo, y a pesar de tratar con estudiantes que pertenecen mayoritariamente a la generación de "nativos digitales", según la revisión conceptual de García, Gértrudix, Durán, Gamonal y Gálvez (2011), el uso del blog ha contribuido a su alfabetización digital, pues no todos ellos están familiarizados con las herramientas y posibilidades de estos recursos. Lo cierto es que la necesidad de editar sus textos e incorporar contenidos multimedia ha ido motivando una competencia digital que los propios estudiantes reconocen utilizar ya para tareas académicas de otras asignaturas o sus prácticas profesionales.

\section{c) El blog amplifica la experiencia de la fase social del aprendizaje}

Aunque no hay un único modo de aprender, durante los últimos años se ha destacado el componente social del aprendizaje que defendiera Vygotsky, frente al sentido más individualista que subrayara Piaget (Zabalza, 2009). Si bien es cierto que es el sujeto quien termina adquiriendo y haciendo suyo el aprendizaje, también lo es que ese resultado sea consecuencia de un contraste e intercambio de información, de opiniones, de ideas previas, de experiencias, que se produce en algún lugar (físico o virtual) y con otros (sean personas o producciones de las personas como un texto, por ejemplo). Desde este punto de vista, la adquisición o consolidación de un aprendizaje en el individuo requiere de una fase anterior comunitaria, de interacción, donde se confronten las ideas previas y se provoque la reconstrucción de los conocimientos, algo especialmente apropiado entre estudiantes universitarios, con un amplio bagaje formativo.

Las características de los blogs, principalmente su interactividad y la posibilidad de enlazar fuentes de información de naturaleza muy diversa, hacen de este recurso un espacio virtual ideal para visibilizar diferentes perspectivas de análisis de los contenidos y provocar el intercambio intelectual (Xie y Sharma, 2005; Zeng y Harris, 2005). Durante estos años, el grupo de innovación ha experimentado que el blog multiplica el número y significado de las interacciones que se producen en el aula debido a los aspectos comentados en los apartados a y b. Es decir, el intercambio no muere con el fin de la lección en el aula, sino que puede continuar vivo en el espacio virtual y resultar más enriquecedor al permitir intervenciones más reflexivas. En este sentido, cuando los profesores del grupo de innovación destacan los aspectos más positivos del blog, es frecuente que señalen el intercambio que posibilitan y los debates constructivos que generan. De hecho, tanto el profesorado como el alumnado coinciden en sus valoraciones:

A través de los blogs utilizados, se han facilitado procesos comunicativos entre los estudiantes y la construcción social de los 
contenidos de la asignatura. Se ha ampliado el espacio de aprendizaje más allá del aula y del horario de clase, ofreciendo un espacio más libre e interactivo de participación a los estudiantes. Los alumnos se sienten más protagonistas, participando con sus posts y comentarios en la (re)elaboración de los contenidos, leyendo los posts y comentarios de los compañeros (P6, 2012-13).

Otra cosa muy positiva es la interacción entre toda la clase con las actividades realizadas. Del profesor se aprende muchísimo, claro está, pero compartiendo con todos nuestros compañeros, hemos conseguido solucionar dudas, aprender cosas nuevas y formas muy distintas de ver las cosas y la asignatura (JJH, 2012-13).

Por otro lado, el blog permite abrir y exponer, si así se desea, la interacción didáctica que profesor y estudiante han mantenido en espacios clásicos como la tutoría, de modo que las orientaciones y los aprendizajes que allí se producen pueden beneficiar también a otros miembros del grupo-clase. Esto es lo que ha sucedido, por ejemplo, en aquellos blogs del tipo D y E utilizados para hacer un seguimiento público y compartido de los trabajos de grupo.

d) El blog proporciona evidencias sobre el progreso de los estudiantes y retroalimenta el proceso de enseñanza-aprendizaje

Entre otras finalidades, el blog puede servir también para tomar el pulso a los progresos de sus estudiantes y reconducir, si fuera necesario, su proceso formativo. El blog puede ayudar a visibilizar el error, la comprensión imperfecta de conceptos o teorías, el grado de desarrollo de sus competencias $\mathrm{y}$, de esta manera, reforzar los aprendizajes necesarios para progresar en la materia con mayor éxito. Varios de los participantes del grupo de innovación refieren esta utilidad del blog. Consideran que "permite al profesor tener un feedback sobre el aprendizaje de los alumnos al poder leer lo que escriben sus alumnos y conocer así cómo entienden los contenidos que se desarrollan en sus clases, pudiendo reajustar sus procesos de enseñanza" (P6, 2012-13). Desde esta perspectiva, más que entender el blog como un instrumento de evaluación formal y explícita, interesa el potencial informativo de este recurso para ser utilizado en una evaluación integrada en las actividades cotidianas del proceso de enseñanza-aprendizaje, tal y como propone Álvarez (2010).

e) El blog estimula el juicio crítico

Participar en un blog exige atender, en primer lugar, a los posts y comentarios sobre el tema en cuestión. Cuando se trata de temas más abiertos, proclives al debate, la intervención de cualquier estudiante ha de conjugar sus propias ideas con la valoración que le merezcan otros puntos de vista y argumentos pues, en caso contrario, no se conservaría ese carácter dialógico del blog al que se refería Fernández (2010). Una buena contribución, por tanto, no puede ser ajena a los puntos principales del debate que se derivan de los comentarios de los participantes. Esto implica movilizar habilidades cognitivas de tipo operacional, fundamentales en la Educación Superior, que permiten 
procesar, organizar y relacionar la información y, en última instancia, interpretarla y enjuiciarla. Como dice esta alumna:

A nivel personal me gusta el poder comentar, que te puedan replicar y volver a contestar. Además sin darme cuenta asiento ciertos conocimientos ya sea por las lecturas extras de bibliografía o simplemente por plasmar aquello de lo que se ha hablado en clase. (AI, 2010-11)

Por otro lado, en varias de las asignaturas participantes en estos proyectos, se ha pedido expresamente a los estudiantes la valoración de las aportaciones o trabajos de sus compañeros. En algún caso se ha dado también la oportunidad de hacer públicas las autoevaluaciones de los estudiantes a través de sus blogs. El hecho de que estos juicios de valor sean públicos y susceptibles de ser comentados o replicados por el resto de estudiantes o por el profesor, obliga a un esfuerzo intelectual mayor para valorar justa y fundadamente el trabajo propio o el de los compañeros.

\section{Aspectos críticos del uso de edublogs en la Educación Superior}

Aunque el balance general del uso de blogs ha resultado positivo, las conclusiones alcanzadas tras los proyectos llevados a cabo por el grupo de innovación también identifican algunos aspectos problemáticos. Entre todos ellos, los principales están relacionados con la participación de los estudiantes y con la gestión del blog por parte del profesor.

En primer lugar, hay que señalar una desigual incorporación del alumnado al uso de este recurso. No todos los estudiantes participan en la misma medida, ni en el número de aportaciones ni en la calidad de la mismas. Al igual que hay profesores que tienen apego por los métodos más tradicionales de enseñanza, hay también estudiantes que mantienen una actitud menos comprometida con un aprendizaje activo y eluden las estrategias que implican mayor protagonismo por su parte. En muchos de estos casos no se produce intervención alguna o la aportación tan solo aspira a dejar huella de su participación en el blog con un grado de elaboración insuficiente. Además puede producirse el fenómeno de la escritura forzada al que se refiere Lara (2005). Alguno de los profesores advirtió también que "el conocimiento de la herramienta introduce una brecha clara entre aquellos que sí que conocen bien el uso (lo que les permite posts y comentarios más ricos en enlaces, vídeos...) y aquellos que están aprendiendo a usarlo sobre la marcha" (P14, 2012-13). Aun así, en el último de los proyectos, donde participó un mayor número de asignaturas y estudiantes, ningún profesor valoró muy negativamente la participación de sus estudiantes en el blog, sólo un 6\% la valoró de forma negativa, un $31 \%$ se mostró neutral y un $63 \%$ del profesorado la valoró positiva o muy positivamente.

En segundo lugar, es frecuente que los edublogs generen un volumen de actividad e información que en ocasiones rebasa la capacidad de supervisión y moderación del profesor. Es cierto que la dedicación de éste depende del uso que se haga del blog y del papel principal o secundario que tenga en la asignatura pero, en cualquier caso, exige 
una atención continua porque, tal y como apunta Fernández (2010), se confía al profesor el cierre de las cuestiones abiertas. De hecho, hay estudiantes que han manifestado que esperan del profesor alguna retroalimentación, ya sea a través del propio blog o en el aula, para despejar la incertidumbre que en ocasiones sienten. Por tanto, si se reconoce la utilidad del blog como recurso de ayuda al aprendizaje y en un contexto de intensificación del puesto de trabajo como el actual, el profesorado debe articular estrategias de supervisión factibles para integrarlo con éxito en sus enseñanzas.

\section{Conclusiones}

Dentro de las TIC, los blogs son un recurso para la conformación de entornos virtuales para la enseñanza y aprendizaje en la Educación Superior. Sin embargo, no hay que suponer que el simple hecho de añadirlos en el desarrollo de las asignaturas asegure el éxito pedagógico o la innovación educativa. Como cualquier recurso o proceso educativo, requiere de una evaluación que permita la valoración crítica del mismo, tanto por parte del profesorado como del alumnado. El propósito de este trabajo fue identificar la contribución de los blogs a los procesos de enseñanzaaprendizaje en la Educación Superior, a partir de las evidencias acumuladas por un grupo de profesores universitarios durante varios proyectos de innovación educativa. De los resultados obtenidos se concluye que:

- El uso de los blogs proporciona continuidad a los aprendizajes iniciados en el aula y facilita el acceso y archivo de fuentes de información complementarias. En aquellos casos en los que el uso del blog está ligado directamente a las actividades del aula, se ha destacado como un recurso muy útil para el seguimiento de la asignatura por parte de aquellos estudiantes que no pueden acudir a clase con regularidad.

- El blog puede representar un estímulo para la participación de estudiantes que no intervienen habitualmente en el "directo" del aula y que, sin embargo, siguen con intensidad la asignatura. Esas intervenciones dan visibilidad a estos estudiantes ante sus profesores y el resto de la clase.

- El blog permite difundir las interacciones didácticas que se producen entre profesores y estudiantes, y entre estos últimos. Esa publicidad extiende el contenido y el beneficio de tales interacciones, más allá de la tutoría o del trabajo en grupos reducidos, a todos los que comparten un mismo proceso formativo.

- El carácter abierto y público del blog promueve la participación reflexiva y responsable de los estudiantes al efectuar sus aportaciones y enjuiciar las de los demás. Además, proporciona evidencias acerca de la evolución de los aprendizajes que permiten coevaluaciones y autoevaluaciones más sólidas.

- Aunque el balance general de la experiencia es altamente positivo, se concluye también que el blog no suscita el mismo grado de implicación entre todos los estudiantes y, por tanto, su contribución al aprendizaje puede resultar desigual. Asimismo, el volumen de la actividad generada en el blog puede sobrepasar la 
capacidad de supervisión del profesor y generar incertidumbre sobre los aprendizajes en juego.

\section{Referencias bibliográficas}

ÁLVAREZ, J. M. (2010). El currículum como marco de referencia para la evaluación educativa. En J. Gimeno (Comp.), Saberes e incertidumbres sobre el currículum (pp. 355-371). Madrid: Morata.

ANTOLÍN, L., MOLINA, P., VILLAMÓN, M., DEVÍS, J. \& PÉREZ-SAMANIEGO, V. (2011). Uso de blogs en ciencias de la actividad física y el deporte. @tic. Revista d'innovació educativa, 7, 12-18.

BUSTOS, A. \& COLL, C. (2010). Los entornos virtuales como espacios de enseñanza y aprendizaje. Una perspectiva psicoeducativa para su caracterización y análisis. Revista Mexicana de Investigación Educativa (RMIE), 15(44), 163-184.

CABERO, J., LÓPEZ, E. \& BALLESTEROS, C. (2009). Experiencias universitarias innovadoras con blogs para la mejora de la praxis educativa en el contexto europeo. Revista de Universidad y Sociedad del Conocimiento (RUSC), 6(2), 114 ..

COLL, C. (2004). Psicología de la educación y prácticas educativas mediadas por las tecnologías de la información y la comunicación. Una mirada constructivista [Separata]. Sinéctica, 25, 1-24.

COLL, C. \& MONEREO, C. (2008). Introducción. En C. Coll y C. Monereo, Psicología de la educación virtual, (pp. 11-16). Madrid: Morata.

COUTINHO, C. (2007). Cooperative learning in higher education using weblogs: A study with undergraduate students of education in Portugal. En World multiconference on systemics, cybernetic and informatics, Vol. 1, (pp. 60-64). Orlando (Estados Unidos). Recuperado de: http://repositorium.sdum.uminho.pt/bitstream/1822/6721/1/Webblogs.pdf, 10 de junio de 2013.

ELLISON, N. B., \& Wu, Y. (2008). Blogging in the classroom: A preliminary exploration of student attitudes and impact on comprehension. Journal of Educational Multimedia and Hypermedia, 17(1), 99-122.

FERNÁNDEZ, A. (2010). Filosofía en el abismo. Apuntes de filosofía mundana para un mundo globalizado (de las excelencias de los blogs educativos frente a las redes sociales). Eikasia. Revista de Filosofía, 32, 197-212.

FRAILE, A. (2002). El seminario colaborativo: una propuesta formativa para el profesorado de Educación Física. Contextos educativos: Revista de educación, 5, 101-122.

GALlEGO, D. J., MARTÍN-CUADRADO, A. M. \& CACHEIRO, M. L. (2010). Aprendiendo de forma colaborativa a través de las herramientas Web 2.0. En R. 
Roig y M. Fiorucci (Eds.), Claves para la investigación en innovación y calidad educativas. La integración de las Tecnologías de la Información y la Comunicación y la Interculturalidad en las aulas, (pp. 163-178). Alcoy \& Roma: Marfil \& Università degli Studi Roma Tre.

GARCÍA, A. \& SUÁREZ, C. (2011). Interacción virtual y aprendizaje cooperativo. Un estudio cualitativo. Revista de Educación, 354, 473-498.

GARCÍA, F., GÉRTRUDIX, F., DURÁN, J. F., GAMONAL, R. \& GÁLVEZ, M. C. (2011). Señas de identidad del "nativo digital”. Una aproximación teórica para conocer las claves de su unicidad. Cuadernos de Documentación Multimedia, 22, 110-127.

LARA, T. (2005). Blogs para educar. Usos de los blogs en una pedagogía constructivista. Telos. Cuadernos de Comunicación e Innovación, 65, 83-96.

MOLINA, P., ANTOLÍN, L. DEVÍS, J., VILLAMÓN, M., PÉREZ-SAMANIEGO, V. \& Valenciano, J. (2013). Els edublogs com eines de participació i seguiment d'activitats acadèmiques complementàries i transversals. IV Trobades d'Innovació Educativa de la Universitat de València. Valencia, enero.

MOLINA, P., ANTOLÍN, L., PÉREZ-SAMANIEGO, V., DEVÍS, J., VILLAMÓN, M. \& Valenciano, J. (2013). Uso de blogs y evaluación continua del aprendizaje del alumnado universitario. EDUTEC, Revista Electrónica de Tecnología Educativa, 43.

MOLINA, P., VALENCIANO, J., VALENCIA-PERIS, A., MUÑOZ, D., MONFORTE, E, MARTOS, D. et al. (2013). Edublogs y aprendizaje colaborativo en la Universidad. En T. Ramiro y M. T. Ramiro, (Comps.), X Foro Internacional sobre la Evaluación de la Calidad de la Investigación y de la Educación Superior (FECIES), (pp. 277-282). Simposio efectuado en el X FECIES, Granada, junio. Recuperado de: http://www.ugr.es/ aepc/XFORO/LIBRORESUMENESXFORO.pdf, 20 de agosto de 2013.

NEIRA, M. R., VILLALUSTRES, L. \& MORAL, E. (2011). Innovaciones con blogs: desarrollo de competencias del futuro maestro y bienestar subjetivo docente. @tic. Revista d'innovació educativa, 9, 14-22.

O’DONNELL, M. (2006). Blogging as pedagogic practice: artefact and ecology. Asia Pacific Media Educator, 17, 5-19.

ORAVEC, J. A. (2003). Blending by Blogging: weblogs in blended learning initiatives. Journal of Educational Media, 28(2-3), 225-233.

SANCHO, J. M. (2008). De TIC a TAC, el difícil tránsito de una vocal. Investigación en la Escuela, 64, 19-30.

SIM, J. W. S. \& HEW, K. F. (2010). The use of weblogs in higher education settings: A review of empirical research. Educational Research Review, 5(2), 151-163. 
VALENCIA-PERIS, A. \& MOLINA, P. (2012). Experiencias de innovación educativa con blogs en la universidad española. En D. Cobos, E. López, A. Jaén, A. Hilario, M. Padilla y L. Molina (dir.), Actas del I Congreso Internacional Virtual sobre innovación pedagógica y praxis educativa Innovagogía 2012, (pp. 757-767). Sevilla: AFOE.

WILLIAMS, J. B. \& JACOBS, J. (2004). Exploring the use of blogs as learning spaces in the higher education sector. Australasian Journal of Educational Technology, 20(2), 232-247.

XIE, Y., KE, F., \& SHARMA, P. (2008). The effect of peer feedback for blogging on college students' reflective learning processes. Internet and Higher Education, 11(1), 18-25.

XIE, Y., \& SHARMA, P. (2005). Students' lived experience of using weblogs in a class: An exploratory study. Association for Educational Communications and Technology. Chicago (IL).

ZABALZA, M. A. (2009). Diseño y desarrollo curricular (11 ${ }^{\mathrm{a}}$ ed.). Madrid: Narcea.

ZENG, X. \& HARRIS, S. T. (2005). Blogging in an online health information technology class. Perspectives in Health Information Management/AHIMA, American Health Information Management Association, 2.

\section{Correspondencia con los autores}

Pere MOLINA ALVENTOSA

Departamento de Educación Física y Deportiva

Facultad de Ciencias de la Actividad Física y el Deporte. Campus de Blasco Ibáñez

C. Gascó Oliag, n 3

46010 Valencia

e-mail: juan.p.molina@uv.es

Javier VALENCIANO VALCÁRCEL

Facultad de Educación de Toledo. Universidad de Castilla La Mancha.

Campus de la Antigua Fábrica de Armas.

Avda. Carlos III, s/n.

45071 Toledo.

e-mail: javier.valenciano@uclm.es

Alexandra VALENCIA-PERIS

Departamento de Didáctica de la Expresión Musical, Plástica y Corporal

Facultad de Magisterio

Campus dels Tarongers

Av. dels Tarongers, $\mathrm{s} / \mathrm{n}$

46022 Valencia

e-mail: alexandra.valencia@uv.es 